INSTITUTE OF FOOD AND RESOURCE ECONOMICS UNIVERSITY OF COPENHAGEN

MSAP Working Paper Series

No. $07 / 2012$

A New Axiomatic Approach to the Evaluation of Population Health

Jens Leth Hougaard

Institute of Food and Resource Economics

University of Copenhagen

Juan D. Moreno-Ternero

Department of Economics

Universida Pablo de Olavide

Lars Peter Østerdal

Department of Business and Economics

University of Southern Denmark

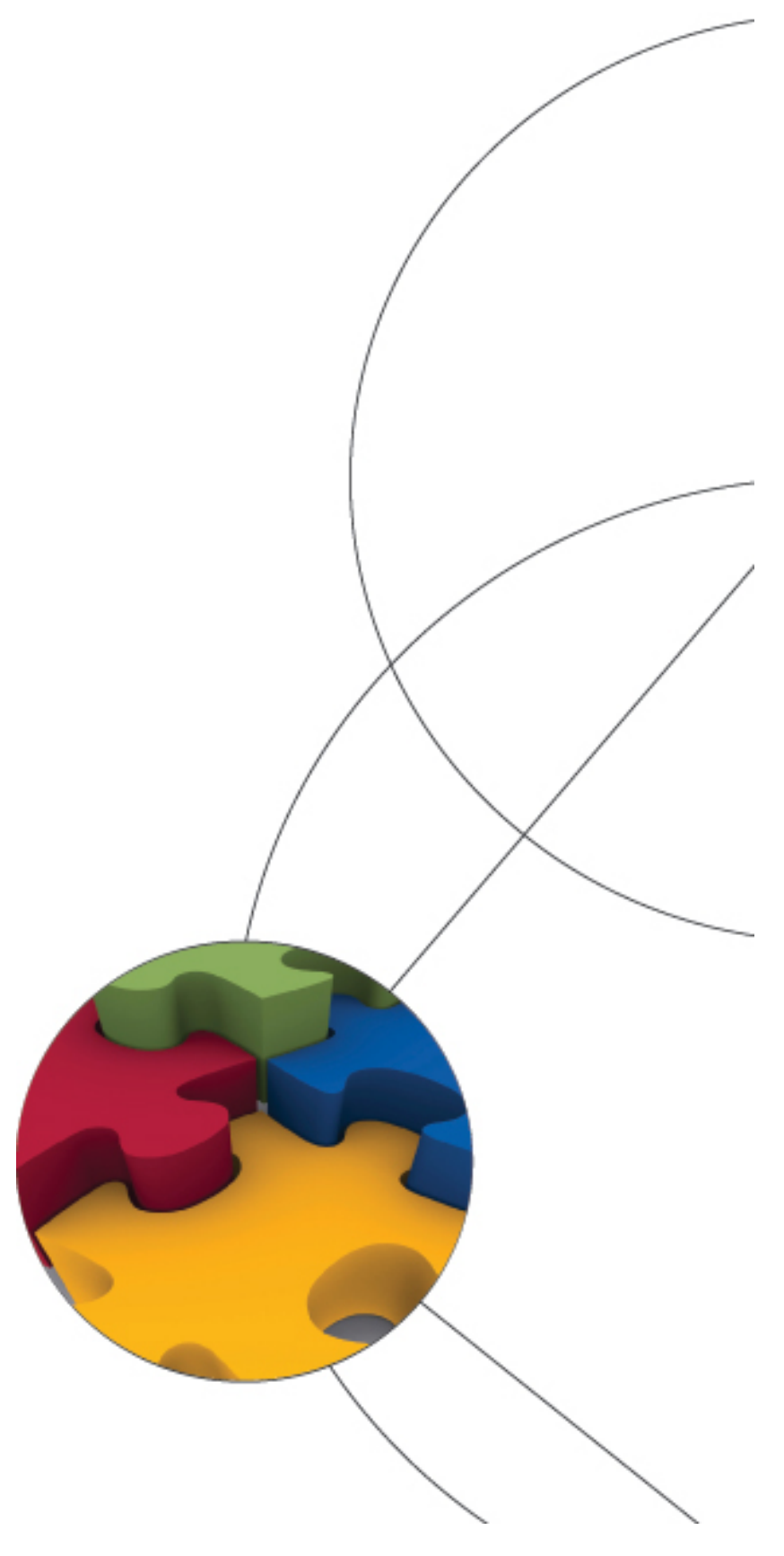




\title{
A new axiomatic approach to the evaluation of population
}

\section{health*}

\author{
Jens Leth Hougaard ${ }^{\dagger}$ Juan D. Moreno-Ternero ${ }^{\ddagger}$ Lars Peter $\varnothing_{\text {sterdal }}^{\mathbf{5}}$
}

March 13, 2012

\begin{abstract}
In this paper we explore the implications of ethical and operational principles for the evaluation of population health. We formalize those principles as axioms for social preferences over distributions of health for a given population. We single out several focal population health evaluation functions, which represent social preferences, as a result of combinations of those axioms. Our results provide rationale for popular theories in health economics (such as the unweighted aggregation of QALYs or HYEs, and generalizations of the two, aimed to capture concerns for distributive justice) without resorting to controversial assumptions over individual preferences.
\end{abstract}

JEL numbers: D63, I10.

Keywords: population health, QALYs, HYEs, axioms.

*We thank Erwin Ooghe, John Roemer, Erik Schokkaert and seminar audiences, as well as conference participants, at Leuven, Malaga, Santiago, and Seville for helpful comments and suggestions. Financial support from the Danish Strategic Research Council, the Spanish Ministry of Science and Innovation (ECO2008-03883, ECO2011-22919) as well as from the Andalusian Department of Economy, Innovation and Science (SEJ-4154, SEJ-5980), via the "FEDER operational program for Andalusia, 2007-2013", is also gratefully acknowledged. Part of this work was done while Juan D. Moreno-Ternero was at the Department of Economic Theory, University of Malaga, and Lars P. Østerdal was at the Department of Economics, University of Copenhagen.

$\dagger$ Institute of Food and Resource Economics, University of Copenhagen.

$\ddagger$ Department of Economics, Universidad Pablo de Olavide, and CORE, Université catholique de Louvain.

$\S$ Department of Business and Economics, University of Southern Denmark.

"Address for correspondence: Lars Peter Østerdal, Department of Business and Economics, University of Southern Denmark, Campusvej 55, 5230 Odense M. Denmark. E-mail: lpro@sam.sdu.dk. 


\section{Introduction}

The objective of health care systems worldwide is to positively affect the health of people. Such an objective places a central role to the definition and measurement of benefit in health care, as well as how it should be distributed in a population. As a result, social scientists, practitioners and health services researchers alike have long been concerned with developing methods to measure the health status of individuals and populations. Attempts to develop appealing quantitative measures to evaluate the health of a population abound in the literature (e.g., Torrance, 1976, 1986; Mehrez and Gafni, 1989; Wagstaff, 1991; Bleichrodt, 1995, 1997; Williams, 1997; Dolan, 1998, 2000; Murray et al., 2002; Bleichrodt et al., 2004; Østerdal, 2005; Fleurbaey and Schokkaert, 2009). The purpose of this paper is to derive some focal measures by means of a new axiomatic approach to the evaluation of population health. Our results provide rationale for popular theories in health economics (and some generalizations of them) without resorting to controversial assumptions over individual preferences.

It is frequently argued that the benefit a patient derives from a particular health care intervention is defined according to two dimensions: quality of life and quantity of life (e.g., Pliskin et al., 1980). We endorse such an assumption in our model. More precisely, we assume that the distribution of health in a population is defined by a collection of duplets, each indicating the status that an agent of the population achieves in the two dimensions: health (quality of life) and time (quantity of life). We refer to those distributions as population health distributions. We departure from earlier contributions within the health economics literature dealing with the evaluation of health distributions, which presume a given relationship between quality and quantity of life at the individual level (e.g., Bleichrodt, 1995, 1997; Østerdal, 2005; Bleichrodt et al., 2004; Doctor et al., 2009, Harvey and Østerdal 2010). In other words, we do not assume from the outset the existence of an index summarizing the information of each duplet. Nevertheless, as it will be shown later, we implicitly derive indices of that sort as a result of combining our axioms.

Our goal in this paper is to single out, as a result of combining several axioms reflecting ethical and operational principles, specific measures to evaluate population health distributions. The key tool of our work is the so-called population health evaluation function ("PHEF" hereafter). A PHEF is a mapping that associates with each population health distribution a real number intended to perform comparisons among them and, hence, reflect social preferences over those distributions. We single out several PHEFs, each reflecting well-known natural views 
in the economic appraisal of health care programmes for resource allocation decisions. The axiomatic characterizations leading to these PHEFs allow us to scrutinize their relative virtues by means of comparing the principles (axioms) that drive each of them.

One of the PHEFs we single out is the so-called (aggregated) time-linear QALY function, which evaluates population health by means of the unweighted aggregation of quality-adjusted life-years (QALYs). In contrast to the literature dealing with this focal and common procedure to aggregate health benefits in economic evaluations of health care, we derive it without imposing any structure on individual preferences over health. As a result, we avoid one of the main criticisms of the QALY measure, namely that it relies on restrictive assumptions over individual preferences (e.g., Loomes and McKenzie, 1989). We also single out the so-called (aggregated) HYE function, which evaluates population health by means of the unweighted aggregation of healthy years equivalents (HYEs). In doing so, we are able to scrutinize further the similarities and differences between these two focal concepts (HYEs and QALYs) in health economics, an aspect that has received considerable attention in the literature (e.g., Mehrez and Gafni, 1989; Culyer and Wagstaff, 1993; Gafni et al., 1993; Bleichrodt, 1995).

Both PHEFs (time-linear QALY and HYE) dismiss a concern for distributive justice in the evaluation process of population health distributions. They simply focus on the aggregate number (of QALYs or HYEs) that a distribution delivers. We also characterize in this paper PHEFs modifying the previous two in order to capture concerns for distributive justice. As it will be shown later, the resulting PHEFs, which are also inspired by classical contributions in the economics literature, are closely connected to other proposals in the literature on health economics.

Finally, it is worth emphasizing that our model only imposes a minimal mathematical structure for the set of available health states. Thus, it is broad enough to partially accommodate other related lines of research, which are popular nowadays, such as the measurement of multidimensional poverty or welfare.

The rest of the paper is organized as follows. In Section 2, we introduce the model and the basic axioms we consider. In Section 3, we introduce and characterize two focal PHEFs. We generalize them, and characterize the ensuing PHEFs, in Section 4. We discuss the results and some possible extensions in Section 5. For a smooth passage, we defer the proofs and provide them in an appendix. 


\section{The preliminaries}

Imagine a policy maker who has to compare distributions of health for a population of fixed size $n \geq 3$. Let us identify the population (society) with the set $N=\{1, \ldots, n\}$. The health of each individual in the population is described by a duplet indicating the level achieved in two parameters: quality of life and quantity of life. Assume that there exists a set of possible health states, $A$, defined generally enough to encompass all possible health states for everybody in the population. We emphasize that $A$ is an abstract set without any particular mathematical structure. ${ }^{1}$ Quantity of life is simply described by a set of nonnegative real numbers, $T \subset \mathbb{R}$. In what follows, we assume that $T=[0,+\infty)$. Formally, let $h_{i}=\left(a_{i}, t_{i}\right) \in A \times T$ denote the health duplet of individual $i .^{2}$ A population health distribution (or, simply, a health profile) $h=\left[h_{1}, \ldots, h_{n}\right]=\left[\left(a_{1}, t_{1}\right), \ldots,\left(a_{n}, t_{n}\right)\right]$ specifies the health duplet of each individual in society. Denote the set of all possible health profiles by $H$, i.e., $H$ is the $n$-Cartesian product of the set $A \times T$. Even though we do not impose a specific mathematical structure on the set $A$, we assume that it contains a specific element, $a_{*}$, referred to as perfect health and which is univocally identified, as a "superior" state, by all agents in the population.

The policy maker's preferences (or social preferences) over health profiles are expressed by a preference relation $\succsim$, to be read as "at least as preferred as". As usual, $\succ$ denotes strict preference and $\sim$ denotes indifference. Assume the relation $\succsim$ is a weak order, i.e., it is complete (for each health profiles $h, h^{\prime}$, either $h \succsim h^{\prime}$, or $h^{\prime} \succsim h$, or both) and transitive (if $h \succsim h^{\prime}$ and $h^{\prime} \succsim h^{\prime \prime}$ then $\left.h \succsim h^{\prime \prime}\right)$.

A population health evaluation function (PHEF) is a real-valued function $P: H \rightarrow \mathbb{R}$. We say that $P$ represents $\succsim$ if

$$
P(h) \geq P\left(h^{\prime}\right) \Leftrightarrow h \succsim h^{\prime}
$$

for each $h, h^{\prime} \in H$. Note that if $P$ represents $\succsim$ then any strictly increasing transformation of $P$ would also do so.

The model just outlined has, at least, two possible interpretations:

For instance, the pair $\left(a_{i}, t_{i}\right)$ could be identifying an agent having a chronic (or "average") health state $a_{i}$ throughout a (remaining) lifetime of length $t_{i}$. In this sense, the model can be used to express a social planner's preference over societies (of the same size) with different

\footnotetext{
${ }^{1} A$ could for instance refer to the resulting multidimensional health states after combining the levels of each dimension of a categorical measure, such as EQ-5D, in all possible ways.

${ }^{2}$ For ease of exposition, we establish the notational convention that $h_{S} \equiv\left(h_{i}\right)_{i \in S}$, for each $S \subset N$.
} 
distributions of the citizens' health states and (remaining) life years.

We may also think of the scenario in which the planner launches an intervention and this intervention results in a health state $a_{i}$ for a period of time $t_{i}$ for each agent $i$ in the society, relative to some status-quo distribution. Different interventions can then be compared on the basis of their resulting distributions of health states and time periods.

\subsection{Basic structural axioms}

We now list a set of basic axioms for social preferences to be considered in this paper. Each of them reflects an ethical or an operational principle. ${ }^{3}$

Our first axiom, anonymity, is a standard formalization of the principle of impartiality, which refers to the fact that ethically irrelevant information is excluded from the evaluation process. In other words, the identity of agents should not matter and the evaluation of the population health should depend only on the list of quality-quantity duplets, not on who holds them. Formally, let $\Pi^{N}$ denote the class of bijections from $N$ into itself. Then,

ANON: $h \sim h_{\pi}$ for each $h \in H$, and each $\pi \in \Pi^{N}$.

The next axiom, separability, underlies the use of incremental analysis in cost-effectiveness analysis, which implies that individuals for whom two treatments yield the same health should not influence the relative evaluation of these treatments (e.g., Gold et al., 1996; Turpcu et al., 2011). More precisely, it says that if the distribution of health in a population changes only for a subgroup of agents in the population, the relative evaluation of the two distributions should only depend on that subgroup. ${ }^{4}$ Formally,

SEP: $\left[h_{S}, h_{N \backslash S}\right] \succsim\left[h_{S}^{\prime}, h_{N \backslash S}\right] \Leftrightarrow\left[h_{S}, h_{N \backslash S}^{\prime}\right] \succsim\left[h_{S}^{\prime}, h_{N \backslash S}^{\prime}\right]$, for each $S \subseteq N$, and $h, h^{\prime} \in H$.

Continuity is the adaptation to our context of a usual axiom. It says that for fixed distributions of health states, the population health ordering is smooth in lifetimes. In spite of being an apparently technical condition, it is also an ethically attractive axiom. It models non-arbitrariness of the social preferences.

\footnotetext{
${ }^{3}$ For an excellent survey on the axiomatic method and many of its applications the reader is referred to Thomson (2001).

${ }^{4}$ The notion of separability has a long tradition of use in models of cooperative decision making (e.g., Moulin, 1988).
} 
CONT: Let $h, h^{\prime} \in H$, and $h^{(k)}$ be a sequence in $H$ such that, for each $i \in N, h_{i}^{(k)}=\left(a_{i}, t_{i}^{(k)}\right) \rightarrow$ $\left(a_{i}, t_{i}\right)=h_{i}$. If $h^{(k)} \succsim h^{\prime}$ for each $k$, then $h \succsim h^{\prime}$.

The next axiom, perfect health superiority, refers to the superiority of the state of perfect health. More precisely, it says that replacing the health status of an agent by that of perfect health, ceteris paribus, cannot worsen the evaluation of the population health. It constitutes the closest approximation in our context to the Pareto principle of optimality.

PHS: For each $h=\left[h_{1}, \ldots, h_{n}\right] \in H$ and $i \in N$, let $h_{i}^{*}=\left(a_{*}, t_{i}\right)$. Then, $\left[h_{i}^{*}, h_{N \backslash\{i\}}\right] \succsim h$.

A somewhat related axiom comes next. Time monotonicity at perfect health says that if each agent is at perfect health, increasing the time dimension is strictly better for society. Formally,

TMPH: If, for each $i \in N, t_{i} \geq t_{i}^{\prime}$, at least one strict, then $\left[\left(a_{*}, t_{1}\right), \ldots,\left(a_{*}, t_{n}\right)\right] \succ\left[\left(a_{*}, t_{1}^{\prime}\right), \ldots,\left(a_{*}, t_{n}^{\prime}\right)\right]$.

The previous two axioms convey some sort of desirability of the perfect health state. The next axiom, however, puts a cap on it. More precisely, non-desirable death states that society (strictly) improves by replacing an agent with perfect health and zero lifetime, with any other agent having a positive lifetime (independently of her health state). Formally,

NDD: For each $h \in H$ and $i \in N$ such that $t_{i}>0, h \succ\left[h_{N \backslash\{i\}},\left(a_{*}, 0\right)\right]$.

The next axiom, social zero condition, also refers to the case of zero lifetime and is reminiscent of a widely used condition for individual utility functions on health (e.g., Bleichrodt et al., 1997; Miyamoto et al., 1998; Østerdal 2005). It says that if an agent gets zero lifetime, then her health state does not influence the social desirability of the health distribution. Formally,

ZERO: For each $h \in H$ and $i \in N$ such that $t_{i}=0$, and $a_{i}^{\prime} \in A, h \sim\left[h_{N \backslash\{i\}},\left(a_{i}^{\prime}, 0\right)\right]$.

In what follows, we refer to the set of axioms introduced above as the basic structural axioms (in short, BASIC).

\section{The characterization of two focal measures}

The most widely employed way of combining the quality of life and quantity of life derived from a particular health care intervention is by means of QALYs. The following PHEF, which we call (aggregated) time-linear $Q A L Y$, evaluates population health distributions by means of 
the unweighted aggregation of individual QALYs in society, or, in other words, by the weighted (through health levels) aggregate time span the distribution yields. Formally,

$$
P^{q}\left[h_{1}, \ldots, h_{n}\right]=P^{q}\left[\left(a_{1}, t_{1}\right), \ldots,\left(a_{n}, t_{n}\right)\right]=\sum_{i=1}^{n} q\left(a_{i}\right) t_{i},
$$

where $q: A \rightarrow[0,1]$ is a function satisfying $0 \leq q\left(a_{i}\right) \leq q\left(a_{*}\right)=1$, for each $a_{i} \in A$.

Our first result says that $P^{q}$ is characterized by the combination of the basic structural axioms described above plus the following specific axiom, time invariance at common health, which refers to the equal value of life gains for persons with common health states. ${ }^{5}$ Formally,

TICH: For each $h \in H, c>0$, and $i, j \in N$, such that $a_{i}=a_{j}$,

$$
\left[\left(a_{i}, t_{i}+c\right),\left(a_{j}, t_{j}\right), h_{N \backslash\{i, j\}}\right] \sim\left[\left(a_{i}, t_{i}\right),\left(a_{j}, t_{j}+c\right), h_{N \backslash\{i, j\}}\right]
$$

Our characterization result comes next.

Theorem 1 The following statements are equivalent:

1. $\succsim$ is represented by a PHEF satisfying (1).

2. $\succsim$ satisfies BASIC and TICH.

An alternative way of combining the quality of life and quantity of life derived from a particular health care intervention is by means of healthy years equivalents (HYEs). The next PHEF, which we call (aggregated) HYE, evaluates population health distributions by means of the aggregation of individuals' HYEs. Formally,

$$
P^{h}\left[h_{1}, \ldots, h_{n}\right]=P^{h}\left[\left(a_{1}, t_{1}\right), \ldots,\left(a_{n}, t_{n}\right)\right]=\sum_{i=1}^{n} f\left(a_{i}, t_{i}\right),
$$

where $f: A \times T \rightarrow T$ is a function indicating the HYEs for each possible individual, i.e., for each $h=\left[h_{1}, \ldots, h_{n}\right]=\left[\left(a_{1}, t_{1}\right), \ldots,\left(a_{n}, t_{n}\right)\right] \in H$,

$$
h \sim\left[\left(a_{*}, f\left(a_{i}, t_{i}\right)\right)_{i \in N}\right]
$$

Our second result characterizes the HYE PHEF by adding to the basic structural axioms a weakening of TICH. More precisely, time invariance at perfect health is the weakening of the

\footnotetext{
${ }^{5}$ Thus, the axiom conveys an absence of lifetime discrimination: an individual is not less worthy of treatment on the sole grounds that she has a longer lifetime. As a matter of fact, the axiom is very similar, although not identical, to the so-called non-age dependence axiom in Østerdal (2005).
} 
above axiom to the case in which the common health state is precisely the perfect health state. Formally,

TIPH: For each $h \in H, c>0$, and $i, j \in N$, such that $a_{i}=a_{j}=a_{*}$,

$$
\left[\left(a_{i}, t_{i}+c\right),\left(a_{j}, t_{j}\right), h_{N \backslash\{i, j\}}\right] \sim\left[\left(a_{i}, t_{i}\right),\left(a_{j}, t_{j}+c\right), h_{N \backslash\{i, j\}}\right]
$$

The characterization result of the HYE PHEF comes next.

Theorem 2 The following statements are equivalent:

1. $\succsim$ is represented by a PHEF satisfying (2).

2. $\succsim$ satisfies BASIC and TIPH.

As shown by the statements of the two theorems, weakening TICH, so that the axiom is restricted to the case in which the common health state is the perfect health state, allows us to move from the (linear) QALY PHEF to the much broader family of (linear) HYE PHEFs.

\section{More general measures}

Both PHEFs highlighted in the previous section amount unweighted aggregation across individuals, an aspect usually criticized in the health economics literature by its lack of concern for distributive justice (e.g., Loomes and McKenzie, 1989; Wagstaff, 1991; Dolan, 1998). We present (and characterize) in this section more general PHEFs, which aim to generalize the above two, in order to capture such a concern.

\subsection{Power measures}

We start with two PHEFs that simply introduce suitable powers in the QALYs (or HYEs) each agent in the population gets. The rationale behind this idea can be traced back to Bergson (e.g., Burk, 1936). In a health economics context, power functions of QALYs were introduced, at an individual level, by Pliskin et al., (1980). ${ }^{6}$ The so-called Bergsonian approach has also been discussed in the health economics literature by Wagstaff (1991) and Williams (1997), among others.

\footnotetext{
${ }^{6}$ See also Bleichrodt and Miyamoto (2003).
} 
Formally, the power $Q A L Y$ PHEF is defined as:

$$
P^{p q}\left[h_{1}, \ldots, h_{n}\right]=P^{p q}\left[\left(a_{1}, t_{1}\right), \ldots,\left(a_{n}, t_{n}\right)\right]=\sum_{i=1}^{n} q\left(a_{i}\right) t_{i}^{\gamma},
$$

where $q: A \rightarrow[0,1]$ is a function satisfying $0 \leq q\left(a_{i}\right) \leq q\left(a_{*}\right)=1$, for each $a_{i} \in A$, and $\gamma \in \mathbb{R}_{++}$ is a positive scalar.

Likewise, the power HYE PHEF is defined as:

$$
P^{p h}\left[h_{1}, \ldots, h_{n}\right]=P^{p h}\left[\left(a_{1}, t_{1}\right), \ldots,\left(a_{n}, t_{n}\right)\right]=\sum_{i=1}^{n} f\left(a_{i}, t_{i}\right)^{\gamma},
$$

where $\gamma \in \mathbb{R}_{++}$is a positive scalar, and $f: A \times T \rightarrow T$ is a function indicating the HYEs for each possible individual, i.e., for each $h=\left[h_{1}, \ldots, h_{n}\right]=\left[\left(a_{1}, t_{1}\right), \ldots,\left(a_{n}, t_{n}\right)\right] \in H$,

$$
h \sim\left[\left(a_{*}, f\left(a_{i}, t_{i}\right)\right)_{i \in N}\right]
$$

As we show next, the above two PHEFs are characterized by combining the following pair of axioms with the basic structural axioms we introduced in Section 2.

The axiom of time scale independence says that evaluations should not depend on the variable we use to measure quantity of life (e.g., days, months, years). More precisely, it says that the ranking of a pair of population health distributions does not reverse when all lifetimes are multiplied by a common positive constant. ${ }^{7}$ The individual level counterpart to this axiom is often referred to in the literature as the constant proportional trade-off assumption (e.g., Pliskin et al., 1980). Previous experimental studies have provided mixed support for such assumption (e.g., Attema and Brouwer, 2010), with mostly person and context-specific violations. Thus, we believe it may be more acceptable for a planner to assume scale independence in time for social preferences rather than for individual preferences.

TSI: For each $c>0$, and $h=\left[\left(a_{i}, t_{i}\right)_{i \in N}\right], h^{\prime}=\left[\left(a_{i}^{\prime}, t_{i}^{\prime}\right)_{i \in N}\right]$

$$
h \succsim h^{\prime} \Rightarrow\left[\left(a_{i}, c t_{i}\right)_{i \in N}\right] \succsim\left[\left(a_{i}^{\prime}, c t_{i}^{\prime}\right)_{i \in N}\right]
$$

A plausible weakening of the previous axiom, time scale independence at perfect health, says that the notion only applies when restricted to perfect health. Formally,

TSIPH: For each $c>0$, and $h=\left[\left(a_{*}, t_{i}\right)_{i \in N}\right], h^{\prime}=\left[\left(a_{*}, t_{i}^{\prime}\right)_{i \in N}\right]$,

$$
h \succsim h^{\prime} \Rightarrow\left[\left(a_{*}, c t_{i}\right)_{i \in N}\right] \succsim\left[\left(a_{*}, c t_{i}^{\prime}\right)_{i \in N}\right] .
$$

\footnotetext{
${ }^{7}$ Østerdal (2005) considers a counterpart of this axiom in his context.
} 
The characterizations of the power versions of the QALY and HYE models come next. ${ }^{8}$

Theorem 3 The following statements are equivalent:

1. $\succsim$ is represented by a PHEF satisfying (3).

2. $\succsim$ satisfies BASIC and TSI.

Theorem 4 The following statements are equivalent:

1. $\succsim$ is represented by a PHEF satisfying (4).

2. ¿ satisfies BASIC and TSIPH.

It is worth noting that the TSI axiom assumes scale independence for arbitrary health states while the TICH axiom assumes time invariance for persons with common health states only. Other than that, the parallelism between Theorems 2 and 4 mimics the parallelism between Theorems 1 and 3 .

\subsection{Other generalizations}

We conclude by considering two generalized (semi-multiplicative) PHEFs. The first one generalizes the time component in the time-linear QALY model so that it enters into the PHEF in an arbitrary (not necessarily linear) way. In that sense, it becomes a sort of hybrid between a time-linear QALY model and the more general structure of the HYE model. A similar type of generalization was axiomatized by Miyamoto et al., (1998) for a model of individual decision making in health. Formally,

$$
P^{q h}\left[h_{1}, \ldots, h_{n}\right]=P^{q h}\left[\left(a_{1}, t_{1}\right), \ldots,\left(a_{n}, t_{n}\right)\right]=\sum_{i=1}^{n} q\left(a_{i}\right) w\left(t_{i}\right),
$$

where $q: A \rightarrow[0,1]$ is a function satisfying $0 \leq q\left(a_{i}\right) \leq q\left(a_{*}\right)=1$, for each $a_{i} \in A$, and $w: T \rightarrow[0,1]$ is a strictly increasing function satisfying $w(0)=0$.

It turns out that this family is singled out when a new axiom, to be defined below, is added to our basic structural axioms. First, we need some prior definitions. A health state

\footnotetext{
${ }^{8}$ One might find natural to restrict both families to those PHEFs corresponding to $\gamma \leq 1$. It turns out that the following characterization results stated in Theorems 3 and 4 could be enriched to characterize the subsequent families, upon adding a Pigou-Dalton transfer axiom stating that a health profile in which two agents at perfect health have different time spans is dominated by the subsequent profile in which those agents keep the same perfect health status, but share a time span equal to the average of the former two.
} 
$a \in A$ is non-degenerate if there exist distributions of life years $\left(t_{i}\right)_{i \in N}$ and $\left(t_{i}^{\prime}\right)_{i \in N}$ such that $\left[\left(a, t_{i}\right)_{i \in N}\right] \nsim\left[\left(a, t_{i}^{\prime}\right)_{i \in N}\right]$. For each $h=\left[\left(a_{i}, t_{i}\right)_{i \in N}\right] \in H$ we say that $t$ is the time egalitarian equivalent if $h \sim\left[\left(a_{i}, t\right)_{i \in N}\right] .{ }^{9}$ The axiom, time egalitarian equivalent invariance for common health states that time egalitarian equivalents are independent of non-degenerate health states when all agents share them.

TEICH: For each $h=\left[\left(a, t_{i}\right)_{i \in N}\right], h^{\prime}=\left[\left(a^{\prime}, t_{i}\right)_{i \in N}\right] \in H$ such that $a, a^{\prime} \in A$ are non-degenerate, their time egalitarian equivalents coincide.

We can now state the formal result.

Theorem 5 The following statements are equivalent:

1. $\succsim$ is represented by a PHEF satisfying (5).

2. ¿ satisfies BASIC and TEICH.

The last PHEF we consider is another natural generalization of the time-linear QALY PHEF, in which individual QALYs are submitted to an arbitrary (but increasing) function before being aggregated. Formally,

$$
P^{g q}\left[h_{1}, \ldots, h_{n}\right]=P^{g q}\left[\left(a_{1}, t_{1}\right), \ldots,\left(a_{n}, t_{n}\right)\right]=\sum_{i=1}^{n} g\left(q\left(a_{i}\right) t_{i}\right),
$$

where $q: A \rightarrow[0,1]$ is a function satisfying $0 \leq q\left(a_{i}\right) \leq q\left(a_{*}\right)=1$, for each $a_{i} \in A$, and $g: \mathbb{R}_{+} \rightarrow \mathbb{R}$ is a strictly increasing function.

This family is singled out when a weaker version of the TSI axiom called time scale independence for common health duplets is added to our basic structural axioms described above. The axiom states that if we have two health profiles with common duplets then the preference between them is independent of a scaling of the life year component. Formally,

TSICD: For each $c>0$, and $h=\left[(a, t)_{i \in N}\right], h^{\prime}=\left[\left(a^{\prime}, t^{\prime}\right)_{i \in N}\right] \in H$,

$$
h \succsim h^{\prime} \Rightarrow\left[(a, c t)_{i \in N}\right] \succsim\left[\left(a^{\prime}, c t^{\prime}\right)_{i \in N}\right]
$$

We now state the formal result.

\footnotetext{
${ }^{9}$ The idea of egalitarian equivalence is deeply rooted in welfare economics. The reader is referred to the excellent monograph by Fleurbaey and Maniquet (2011) for a recent account of the many applications that have been made of this idea in related contexts.
} 
Theorem 6 The following statements are equivalent:

1. $\succsim$ is represented by a PHEF satisfying (6).

2. $\succsim$ satisfies BASIC and TSICD.

\section{Discussion}

We have presented in this paper a new axiomatic approach to the evaluation of population health. Contrary to most of the previous axiomatic work within the field, we have considered a model in which no considerations about individual preferences over health have been made. In doing so, we bypass a recurrent criticism regarding the difficulty of assessing individual preferences over health profiles (e.g., Dolan, 2000). ${ }^{10}$ We have shown that a framework in which information on individual preferences over health is not available (either for practical or ethical reasons) still allows to make sound decisions over the evaluation of population health.Somewhat related, even though we assume (as customary) that individual health is determined by two dimensions (quality and quantity) we do not presume that individuals evaluate them in a specific given way. In other words, as opposed to earlier axiomatic contributions on the evaluation of health profiles, we do not assume that the health of an individual is summarized by a number (to be interpreted as the number of QALYs, life years, or "health utility" experienced by a person), but by a duplet referring to the two dimensions.

We have characterized two focal representations of social preferences over population health distributions; namely, the time-linear QALY and HYE representations. They translate into our context two of the most well-known and employed techniques to measure the benefits of health interventions in cost-utility analyses. Those techniques were initially considered as polars, although some of their differences and similar aspects were addressed (e.g., Mehrez and Gafni, 1989; Culyer and Wagstaff, 1993; Gafni et al., 1993; Bleichrodt, 1995; Wakker, 1996). We have seen in this paper that they share a solid common ground. To wit, their characterizations share many axioms (anonymity, separability, continuity, perfect health superiority, time monotonicity at perfect health, non-desirable death, and the social zero condition) and differ only in one (the time-linear QALY requires time invariance at common health, whereas the HYE only requires time invariance at perfect health).

\footnotetext{
${ }^{10}$ Furthermore, it has been recently argued in the so-called Global Burden of Diseases, Injuries, and Risk Factors Study that person trade-off (rather than individual preference) information should provide the basis for making priorities.
} 
It is worth mentioning that our aim has been to derive specific representations of social preferences over population health distributions as a result of combining several ethical and operational axioms. We believe our axioms are compelling from a normative viewpoint (although, obviously, some to a higher extent than others). The positive appeal of our axioms has not been tested in this paper. Nevertheless, there exists a wide variety of experimental contributions testing empirically some of the principles (or related ideas) over which our axioms rely in related contexts (e.g., Spencer, 2003; Doctor et al., 2004; Turpcu et al., 2011). The test of the precise axioms we use in our specific context is left for further research.

As mentioned above, our axiomatic characterizations permit to scrutinize the virtues and shortcomings of the PHEFs we single out by means of comparing the principles (axioms) that drive each of them. In particular, we have seen that the time-linear QALY and HYE model rely on the principles of time invariance at common (or perfect) health, whose normative appeal is controversial. This is therefore questioning the normative appeal of these focal models themselves or, at the very least, some of the frameworks over which they are applied.

A relevant aspect of our work has been to impose a minimal mathematical structure for the set of available health states. This enables future extensions linking our framework to other popular lines of research nowadays, such as the measurement of multidimensional income inequality, poverty or welfare, or the evaluation of population health in a context in which health states worse than dead are allowed. For instance, we could assume that $a_{i}$ reflects both the quantity and quality of $i$ 's health state, whereas $t_{i}$ reflects the income of agent $i$. In doing so, the social planner's preference would not just be defined over health distributions, but rather over a proxy of welfare distributions, provided one interprets welfare as a combination of health and income. ${ }^{11}$

To conclude, we acknowledge that our analysis has not dealt with uncertainty. More precisely, we have considered a formulation of the population health evaluation problem which contains no explicit element of risk, and in which we obtain characterizations of population health evaluation functions without assumptions on the social planner's (or individuals') risk attitudes. This allows us to escape from the usual critiques of the expected utility theory that are normally considered in the health economics literature (e.g., Bleichrodt et al., 2001). It is anyhow left for future research to study a counterpart version of our model dealing with uncertainty.

\footnotetext{
${ }^{11}$ This interpretation is reminiscent of the approach taken by Fleurbaey and Shokkaert (2009).
} 


\section{Appendix. Proofs}

Before proving our theorems, we start with two preliminary results. The first one says that our basic structural axioms imply the following property of healthy years equivalence, which says that any population health distribution has a socially equivalent one in which the health outcome of one (and only one) agent is replaced by that of full health, for some quantity of time. ${ }^{12}$ Formally,

HYE: For each $h \in H$ and $i \in N$ there exists $t_{i}^{*}$ such that $h \sim\left[h_{N \backslash\{i\}},\left(a_{*}, t_{i}^{*}\right)\right]$.

Note that the HYE axiom only postulates that, for each agent and health profile, a "healthy years equivalent" exists, but not how it should be determined as a function of the health profile.

Lemma 1 If $\succsim$ satisfies BASIC, then it also satisfies $H Y E$.

Proof. Let $i \in N$ and $h=\left[h_{N \backslash\{i\}},\left(a_{i}, t_{i}\right)\right] \in H$. Our aim is to show that there exists $t_{i}^{*} \geq 0$ such that

$$
h \sim\left[h_{N \backslash\{i\}},\left(a_{*}, t_{i}^{*}\right)\right] .
$$

If $t_{i}=0$, it follows, by $Z E R O$, that $t_{i}^{*}=0$. Otherwise, suppose that $t_{i}>0$, and, by contradiction, that such $t_{i}^{*}$ does not exist. Then, $T=A \cup B$, where,

$$
A=\left\{s \in T \mid h \succ\left[h_{N \backslash\{i\}},\left(a_{*}, s\right)\right]\right\}
$$

and

$$
B=\left\{s \in T \mid\left[h_{N \backslash\{i\}},\left(a_{*}, s\right)\right] \succ h\right\} .
$$

By NDD, $0 \in A$ and, thus, $A \neq \varnothing$. Similarly, by PHS and TMPH, $t_{i}+\varepsilon \in B$, for some $\varepsilon>0$, and thus $B \neq \varnothing$. By CONT, $A$ and $B$ are open sets relative to $T$. As $A \cap B=\varnothing$, it would follow that $T$ is not a connected set, a contradiction.

Finally, $t_{i}^{*}$ is well defined and uniquely determined by SEP and TMPH.

Our second preliminary result provides the characterization derived from combining our basic structural axioms. Formally, separable PHEFs are defined as follows:

$$
P^{s}\left[h_{1}, \ldots, h_{n}\right]=P^{s}\left[\left(a_{1}, t_{1}\right), \ldots,\left(a_{n}, t_{n}\right)\right]=\sum_{i=1}^{n} g\left(f\left(a_{i}, t_{i}\right)\right),
$$

where $f: A \times T \rightarrow \mathbb{R}_{+}$and $g: \mathbb{R}_{+} \rightarrow \mathbb{R}$ are such that:

\footnotetext{
${ }^{12}$ This notion can be traced back to Mehrez and Gafni (1989) who propose it as a plausible way to reflect patient's preferences over health.
} 
- $f$ is continuous with respect to its second variable,

- $0 \leq f\left(a_{i}, t_{i}\right) \leq t_{i}$, for each $\left(a_{i}, t_{i}\right) \in A \times T$,

- $h \sim\left[\left(a_{*}, f\left(a_{i}, t_{i}\right)\right)_{i \in N}\right]$, for each $h=\left[\left(a_{1}, t_{1}\right), \ldots,\left(a_{n}, t_{n}\right)\right] \in H$, and

- $g$ is a strictly increasing and continuous function.

Lemma 2 The following statements are equivalent:

1. $\succsim$ is represented by a PHEF satisfying (7).

2. $\succsim$ satisfies BASIC.

Proof. We focus on the non-trivial implication. Formally, assume $\succsim$ satisfies BASIC. By Lemma 1, $\succsim$ satisfies HYE too. Let $h \in H$. By HYE, for each $i \in N$, there exists $t_{i}^{*}$ such that $h \sim\left[h_{N \backslash\{i\}},\left(a_{*}, t_{i}^{*}\right)\right]$. By SEP, $t_{i}^{*}$ only depends on $\left(a_{i}, t_{i}\right)$ (and, thus, is independent of the remaining duplets of the profile). Thus, for each $i=1, \ldots, n$, let $f_{i}: A \times T \rightarrow \mathbb{R}$ be defined such that $f_{i}\left(a_{i}, t_{i}\right)=t_{i}^{*}$, for each $\left(a_{i}, t_{i}\right) \in A \times T$. By ANON,$f_{i}(\cdot, \cdot) \equiv f_{j}(\cdot, \cdot) \equiv f(\cdot, \cdot)$, for each $i, j \in N$. By TMPH and PHS, $0 \leq f\left(a_{i}, t_{i}\right) \leq t_{i}$, for each $\left(a_{i}, t_{i}\right) \in A \times T$ and, by CONT, $f$ is a continuous function with respect to its second variable. Furthermore,

$$
h \sim\left[\left(a_{*}, f\left(a_{i}, t_{i}\right)\right)_{i \in N}\right]
$$

which implicitly says that social preferences only depend on the profile of healthy years equivalents, and, by CONT, they do so continuously. It also follows that the range of $f$ is a connected subset of $\mathbb{R}$. By Theorem 3 in Debreu (1960), there exists a strictly increasing and continuous function $g: \mathbb{R}_{+} \rightarrow \mathbb{R}$ such that

$$
h \succsim h^{\prime} \Longleftrightarrow \sum_{i=1}^{n} g\left(f\left(a_{i}, t_{i}\right)\right) \geq \sum_{i=1}^{n} g\left(f\left(a_{i}^{\prime}, t_{i}^{\prime}\right)\right),
$$

which concludes the proof.

\subsection{Proof of Theorem 1}

We focus on the non-trivial implication, i.e., $2 \rightarrow 1$. Formally, assume $\succsim$ satisfies BASIC and TICH. Then, by Lemma $2, \succsim$ can be represented by a PHEF satisfying (7). Without loss of generality, let us assume that $g(f(\hat{a}, 0))=0$ for some $\hat{a} \in A$. 
Let $\bar{a} \in A$ be an arbitrary health state. Then, by iterated application of TICH, and the transitivity of $\succsim$,

$$
\sum_{i=1}^{n} g\left(f\left(\bar{a}, t_{i}\right)\right)=g\left(f\left(\bar{a}, \sum_{i=1}^{n} t_{i}\right)\right)+(n-1) g(f(\bar{a}, 0)) .
$$

By ZERO, $g(f(\bar{a}, 0))=g(f(\hat{a}, 0))=0$. Thus,

$$
\sum_{i=1}^{n} g\left(f\left(\bar{a}, t_{i}\right)\right)=g\left(f\left(\bar{a}, \sum_{i=1}^{n} t_{i}\right)\right) .
$$

It then follows that $g(f(\bar{a}, \cdot))$ satisfies

$$
g\left(f\left(\bar{a}, t_{1}+t_{2}\right)\right)=g\left(f\left(\bar{a}, t_{1}\right)\right)+g\left(f\left(\bar{a}, t_{2}\right)\right)
$$

for any $t_{1}, t_{2} \in T$, which is precisely one of Cauchy's canonical functional equations. As $g(f(\bar{a}, \cdot))$ is a continuous function, it follows that the unique solutions to such equation are the linear functions (e.g., Aczel, 2006; page 34). More precisely, there exists a function $\hat{q}: A \rightarrow \mathbb{R}$ such that

$$
g(f(\bar{a}, t))=\hat{q}(\bar{a}) t
$$

for each $\bar{a} \in A$, and $t \in T$. By PHS and TMPH, it follows that $0 \leq \hat{q}\left(a_{*}\right)$ and $\hat{q}\left(a_{i}\right) \leq \hat{q}\left(a_{*}\right)$, for each $a_{i} \in A$. We now show that $\hat{q}\left(a_{i}\right) \geq 0$, for each $a_{i} \in A$. To do so, let $a_{i} \in A$. By Lemma 1 , there exists $t_{i} \in T$ such that $\hat{q}\left(a_{i}\right)=g\left(f\left(a_{i}, 1\right)\right)=g\left(f\left(a_{*}, t_{i}\right)\right)$. By TMPH and ZERO, $g\left(f\left(a_{*}, t_{i}\right)\right) \geq g\left(f\left(a_{*}, 0\right)\right)=g(f(\hat{a}, 0))=0$. Altogether, it says that $\hat{q}\left(a_{i}\right) \geq 0$. To conclude, let $q: A \rightarrow \mathbb{R}$ be such that $q(a)=\frac{\hat{q}(a)}{\hat{q}\left(a_{*}\right)}$, for each $a \in A$. Then, it follows from the above that $0 \leq q\left(a_{i}\right) \leq q\left(a_{*}\right)=1$, for each $a_{i} \in A$. Thus, $\succsim$ is represented by a PHEF satisfying (1), as desired.

\subsection{Proof of Theorem 2}

We focus on the non-trivial implication, i.e., $2 \rightarrow 1$. Formally, assume $\succsim$ satisfies BASIC and TIPH. Then, by Lemma $2, \succsim$ can be represented by a PHEF satisfying (7). Let $h=$ $\left[\left(a_{1}, t_{1}\right), \ldots,\left(a_{n}, t_{n}\right)\right] \in H$, and $h^{\prime}=\left[\left(a_{1}^{\prime}, t_{1}^{\prime}\right), \ldots,\left(a_{n}^{\prime}, t_{n}^{\prime}\right)\right] \in H$. Then, by iterated application of TIPH, and the transitivity of $\succsim$,

$$
h \succsim h^{\prime} \Longleftrightarrow\left[\left(a_{*}, \sum_{i \in N} f\left(a_{i}, t_{i}\right)\right),\left(a_{*}, 0\right)_{k \in N \backslash\{i\}} \succsim\left[\left(a_{*}, \sum_{i \in N} f\left(a_{i}^{\prime}, t_{i}^{\prime}\right)\right),\left(a_{*}, 0\right)_{k \in N \backslash\{i\}}\right] .\right.
$$

By PHS, and the transitivity of $\succsim$,

$$
h \succsim h^{\prime} \Longleftrightarrow \sum_{i \in N} f\left(a_{i}, t_{i}\right) \geq \sum_{i \in N} f\left(a_{i}^{\prime}, t_{i}^{\prime}\right)
$$

as desired. 


\subsection{Proof of Theorem 3}

We focus on the non-trivial implication, i.e., $2 \rightarrow 1$. Formally, assume $\succsim$ satisfies BASIC and TSI. Then, by Lemma 2 , can be represented by a PHEF satisfying (7).

Step 1. We claim that for each $(a, t),\left(a^{\prime}, t^{\prime}\right) \in A \times T$, and $c>0$,

$$
f(a, t) \geq f\left(a^{\prime}, t^{\prime}\right) \Longleftrightarrow f(a, c t) \geq f\left(a^{\prime}, c t^{\prime}\right) .
$$

Indeed, let $h=\left[\left(a_{1}, t_{1}\right), \ldots,\left(a_{n}, t_{n}\right)\right] \in H$ and $c>0$. Denote $h^{c}=\left[\left(a_{1}, c t_{1}\right), \ldots,\left(a_{n}, c t_{n}\right)\right]$ and let $(a, t),\left(a^{\prime}, t^{\prime}\right) \in A \times T$. By $(7)$,

$$
\left[(a, t), h_{N \backslash\{i\}}\right] \succsim\left[\left(a^{\prime}, t^{\prime}\right), h_{N \backslash\{i\}}\right] \Longleftrightarrow f(a, t) \geq f\left(a^{\prime}, t^{\prime}\right)
$$

and

$$
\left[(a, c t), h_{N \backslash\{i\}}^{c}\right] \succsim\left[\left(a^{\prime}, c t^{\prime}\right), h_{N \backslash\{i\}}^{c}\right] \Longleftrightarrow f(a, c t) \geq f\left(a^{\prime}, c t^{\prime}\right)
$$

By TSI,

$$
\left[(a, t), h_{N \backslash\{i\}}\right] \succsim\left[\left(a^{\prime}, t^{\prime}\right), h_{N \backslash\{i\}}\right] \Longleftrightarrow\left[(a, c t), h_{N \backslash\{i\}}^{c}\right] \succsim\left[\left(a^{\prime}, c t^{\prime}\right), h_{N \backslash\{i\}}^{c}\right] .
$$

The transitivity of $\succsim$ concludes.

Step 2. We now claim the following. Let $\bar{q}: A \rightarrow \mathbb{R}$ be such that $\bar{q}(a)=f(a, 1)$, for each $a \in A$. Then,

$$
f(a, t) \geq f\left(a^{\prime}, t^{\prime}\right) \Longleftrightarrow \bar{q}(a) t \geq \bar{q}\left(a^{\prime}\right) t^{\prime},
$$

for each $(a, t),\left(a^{\prime}, t^{\prime}\right) \in A \times T$.

Indeed, by definition, $f\left(a_{i}, 1\right)=f\left(a_{*}, \bar{q}\left(a_{i}\right)\right)$. By Step 1,

$$
f(a, t)=f\left(a^{\prime}, t^{\prime}\right) \Longleftrightarrow f(a, c t)=f\left(a^{\prime}, c t^{\prime}\right) .
$$

Thus, $f\left(a_{i}, t_{i}\right)=\bar{q}\left(a_{i}\right) t_{i}$, as desired.

Step 3. We claim there exists $\gamma>0$ such that, for each $h=\left[\left(a_{1}, t_{1}\right), \ldots,\left(a_{n}, t_{n}\right)\right] \in H$, and $h^{\prime}=\left[\left(a_{1}^{\prime}, t_{1}^{\prime}\right), \ldots,\left(a_{n}^{\prime}, t_{n}^{\prime}\right)\right] \in H$,

$$
h \succsim h^{\prime} \Longleftrightarrow \sum_{i=1}^{n}\left(\bar{q}\left(a_{i}\right) t_{i}\right)^{\gamma} \geq \sum_{i=1}^{n}\left(\bar{q}\left(a_{i}^{\prime}\right) t_{i}^{\prime}\right)^{\gamma} .
$$

Let $P$ denote the PHEF defined by ${ }^{13}$

$$
P\left[h_{1}, \ldots, h_{n}\right]=P\left[\left(a_{1}, t_{1}\right), \ldots,\left(a_{n}, t_{n}\right)\right]=\sum_{i=1}^{n} g\left(\bar{q}\left(a_{i}\right) t_{i}\right) .
$$

\footnotetext{
${ }^{13}$ The proof of this step follows closely the argument of the proof of Theorem 8 in Østerdal (2005).
} 
By Step $2, f(\cdot, \cdot)$ is a monotonic transformation of the function $\tau: A \times T \rightarrow \mathbb{R}$ defined by $\tau(a, t)=\bar{q}(a) t$, for each $(a, t) \in A \times T$. Then, by (7), $P$ represents $\succsim$. By TSI,

$$
\sum_{i=1}^{n} g\left(\bar{q}\left(a_{i}\right) t_{i}\right) \geq \sum_{i=1}^{n} g\left(\bar{q}\left(a_{i}^{\prime}\right) t_{i}^{\prime}\right) \Longleftrightarrow \sum_{i=1}^{n} g\left(\bar{q}\left(a_{i}\right) c t_{i}\right) \geq \sum_{i=1}^{n} g\left(\bar{q}\left(a_{i}^{\prime}\right) c t_{i}^{\prime}\right),
$$

for each $h=\left[\left(a_{1}, t_{1}\right), \ldots,\left(a_{n}, t_{n}\right)\right] \in H, h^{\prime}=\left[\left(a_{1}^{\prime}, t_{1}^{\prime}\right), \ldots,\left(a_{n}^{\prime}, t_{n}^{\prime}\right)\right] \in H$ and $c>0$.

By Bergson and Samuelson (e.g., Burk, 1936; Samuelson, 1965), there are only three possible functional forms for $P$, when restricted to the domain of health profiles with strictly positive time spans:

- $P\left[h_{1}, \ldots, h_{n}\right]=P\left[\left(a_{1}, t_{1}\right), \ldots,\left(a_{n}, t_{n}\right)\right]=\sum_{i=1}^{n} \alpha_{i}\left(\bar{q}\left(a_{i}\right) t_{i}\right)^{\gamma}$,

- $P\left[h_{1}, \ldots, h_{n}\right]=P\left[\left(a_{1}, t_{1}\right), \ldots,\left(a_{n}, t_{n}\right)\right]=-\sum_{i=1}^{n} \alpha_{i}\left(\bar{q}\left(a_{i}\right) t_{i}\right)^{\delta}$,

- $P\left[h_{1}, \ldots, h_{n}\right]=P\left[\left(a_{1}, t_{1}\right), \ldots,\left(a_{n}, t_{n}\right)\right]=\sum_{i=1}^{n} \alpha_{i} \log \left(\bar{q}\left(a_{i}\right) t_{i}\right)$,

for some $\gamma>0, \delta<0$ and $\alpha_{i}>0$ for each $i \in N$.

It is straightforward to show that the last two functional forms cannot be continuously extended to the whole domain $H$, in which zero time spans are allowed. By ANON, $\alpha_{i}=\alpha_{j}$ for each $i, j \in N$. Finally, let $q: A \rightarrow \mathbb{R}$ be such that $q(a)=\bar{q}(a)^{\gamma}$, for each $a \in A$. Altogether, we have that $P=P^{p q}$, as desired.

\subsection{Proof of Theorem 4}

We focus on the non-trivial implication, i.e., $2 \rightarrow 1$. Formally, assume $\succsim$ satisfies BASIC and TSIPH. Then, by Lemma $2, \succsim$ can be represented by a PHEF satisfying (7).

By TSIPH,

$$
\sum_{i=1}^{n} g\left(f\left(a_{i}, t_{i}\right)\right) \geq \sum_{i=1}^{n} g\left(f\left(a_{i}^{\prime}, t_{i}^{\prime}\right)\right) \Longleftrightarrow \sum_{i=1}^{n} g\left(f\left(a_{i}, c t_{i}\right)\right) \geq \sum_{i=1}^{n} g\left(f\left(a_{i}^{\prime}, c t_{i}^{\prime}\right)\right),
$$

for each $h=\left[\left(a_{1}, t_{1}\right), \ldots,\left(a_{n}, t_{n}\right)\right] \in H, h^{\prime}=\left[\left(a_{1}^{\prime}, t_{1}^{\prime}\right), \ldots,\left(a_{n}^{\prime}, t_{n}^{\prime}\right)\right] \in H$ and $c>0$.

Let $P$ be a PHEF representing $\succsim$. As in the proof of Theorem 3, by Bergson and Samuelson, ANON, and the requirement that $P$ represents $\succsim$ in the whole domain $H$, it follows that

$$
P\left[h_{1}, \ldots, h_{n}\right]=P\left[\left(a_{1}, t_{1}\right), \ldots,\left(a_{n}, t_{n}\right)\right]=\sum_{i=1}^{n}\left(f\left(a_{i}, t_{i}\right)\right)^{\gamma},
$$

for some $\gamma>0$, and for each $h=\left[\left(a_{1}, t_{1}\right), \ldots,\left(a_{n}, t_{n}\right)\right] \in H$, which concludes the proof. 


\subsection{Proof of Theorem 5}

We focus on the non-trivial implication, i.e., $2 \rightarrow 1$. Formally, assume $\succsim$ satisfies BASIC and TEICH. Then, by Lemma $2, \succsim$ can be represented by a PHEF satisfying (7).

Let $w: T \rightarrow \mathbb{R}_{+}$be such that $w(t)=f\left(a_{*}, t\right)$, for each $t \in T .{ }^{14}$ By ZERO, $w(0)=0$. Let $a$ be an arbitrary non-degenerate health state and consider the resulting health profile $h \in H$ in which a fraction $p$ of the population experience $a$ for a period $t_{1}$ whereas the rest experience it for a period $t_{2} \neq t_{1}$. Let $t^{*}\left(t_{1}, t_{2}\right)$ be the time egalitarian equivalent. By (7), $p f\left(a, t_{1}\right)+(n-p) f\left(a, t_{2}\right)=n f\left(a, t^{*}\left(t_{1}, t_{2}\right)\right)$, and, thus, $\frac{p}{n} f\left(a, t_{1}\right)+\left(1-\frac{p}{n}\right) f\left(a, t_{2}\right)=f\left(a, t^{*}\left(t_{1}, t_{2}\right)\right)$. By TEICH we have $\frac{p}{n} w\left(t_{1}\right)+\left(1-\frac{p}{n}\right) w\left(t_{2}\right)=w\left(t^{*}\left(t_{1}, t_{2}\right)\right)$. As this holds for any $t_{1}, t_{2} \geq 0, t_{1} \neq t_{2}$, the function $f(a, t)$, for fixed $a$, is an affine transformation of each $w(t)$. Hence, there are functions $\alpha: A \rightarrow \mathbb{R}_{+}, \beta: A \rightarrow \mathbb{R}_{+}$, such that

$$
f(a, t)=\alpha(a) w(t)+\beta(a)
$$

By ZERO we get $\beta(a)=0$ for each $a \in A$. By HYE, $\alpha(a)>0$ for each non-degenerate state $a \in A$. Then, define the function $q: A \rightarrow \mathbb{R}_{+}$by $q(a)=\alpha(a)$ for each non-degenerate state $a \in$ $A$ and $q(a)=0$ otherwise. As a result, $f(a, t)=q(a) w(t)$. By TMPH, $w$ is strictly increasing. By HYE, $q(a) \geq 0$ for each $a \in A$. Finally, by TMPH, and the fact that $h \sim\left[\left(a_{*}, f\left(a_{i}, t_{i}\right)\right)_{i \in N}\right]$, we obtain that $q(a) \leq q\left(a_{*}\right)=1$, for each $a \in A$, which concludes the proof.

\subsection{Proof of Theorem 6}

We focus on the non-trivial implication, i.e., $2 \rightarrow 1$. Formally, assume $\succsim$ satisfies BASIC and TSICD. Then, by Lemma $2, \succsim$ can be represented by a PHEF satisfying (7).

Step 1. We claim that for each $(a, t),\left(a^{\prime}, t^{\prime}\right) \in A \times T$, and $c>0$,

$$
f(a, t) \geq f\left(a^{\prime}, t^{\prime}\right) \Longleftrightarrow f(a, c t) \geq f\left(a^{\prime}, c t^{\prime}\right) .
$$

Indeed, let $h=[(a, t), \ldots,(a, t)], h^{\prime}=\left[\left(a^{\prime}, t^{\prime}\right), \ldots,\left(a^{\prime}, t^{\prime}\right)\right] \in H$ and $c>0$. Denote $h_{c}=$ $[(a, c t), \ldots,(a, c t)]$ and $h_{c}^{\prime}=\left[\left(a^{\prime}, c t^{\prime}\right), \ldots,\left(a^{\prime}, c t^{\prime}\right)\right]$. By $(7)$,

$$
h \succsim h^{\prime} \Longleftrightarrow f(a, t) \geq f\left(a^{\prime}, t^{\prime}\right)
$$

and

$$
h_{c} \succsim h_{c}^{\prime} \Longleftrightarrow f(a, c t) \geq f\left(a^{\prime}, c t^{\prime}\right)
$$

\footnotetext{
${ }^{14}$ Part of the ensuing proof parallels an argument in the proof of Theorem 3.1 in Miyamoto et al., (1998).
} 
By TSICD, the claim follows.

Step 2. Let $\bar{q}: A \rightarrow \mathbb{R}$ be such that $\bar{q}(a)=f(a, 1)$, for each $a \in A$. We claim that

$$
f(a, t) \geq f\left(a^{\prime}, t^{\prime}\right) \Longleftrightarrow \bar{q}(a) t \geq \bar{q}\left(a^{\prime}\right) t^{\prime}
$$

for each $(a, t),\left(a^{\prime}, t^{\prime}\right) \in A \times T$.

The proof is similar to that of Step 2 in the proof of Theorem 3.

By Step $2, f(\cdot, \cdot)$ is a monotonic transformation of the function $\tau: A \times T \rightarrow \mathbb{R}$ defined by $\tau(a, t)=\bar{q}(a) t$, for each $(a, t) \in A \times T$. Then, by the above, $P^{g q}$ represents $\succsim$, as desired.

\section{References}

[1] Aczel, J., 2006. Lectures on functional equations and their applications. Dover.

[2] Attema A., Brouwer W., 2010. On the (not so) constant proportional trade-off. Quality of Life Research 19, 489-497.

[3] Bleichrodt, H., 1995. QALYs and HYEs: Under what conditions are they equivalent? Journal of Health Economics 14, 17-37.

[4] Bleichrodt, H., 1997. Health utility indices and equity considerations. Journal of Health Economics 16, 65-91.

[5] Bleichrodt, H., Diecidue, E., Quiggin, J., 2004. Equity Weights in the Allocation of Health Care: The Rank-Dependent QALY Model. Journal of Health Economics 23, 157-171.

[6] Bleichrodt, H., Miyamoto, J., 2003. A Characterization of quality-adjusted life-years under cumulative prospect theory. Mathematics of Operations Research 28, 181-193

[7] Bleichrodt, H., Pinto, J., Wakker, P., 2001. Making Descriptive Use of Prospect Theory to Improve the Prescriptive Use of Expected Utility. Management Science 47, 1498-1514.

[8] Bleichrodt, H., Wakker, P., Johannesson, M., 1997. Characterizing QALYs by risk neutrality. Journal of Risk and Uncertainty 15, 107-114.

[9] Burk, A., 1936. Real income, expenditure proportionality, and Frisch's new methods of measuring marginal utility. Review of Economic Studies 4, 33-52. 
[10] Culyer, A., Wagstaff, A., 1993. QALYs versus HYEs, Journal of Health Economics 12, 311-323.

[11] Debreu, G., 1960, Topological methods in cardinal utility theory, in K. Arrow, S. Karlin and P. Suppes, (Eds.), Mathematical methods in the social sciences. Stanford University Press.

[12] Doctor, J., Bleichrodt, H., Miyamoto, J., Temkin, N., Dikmen, S., 2004. A New and More Robust Test of QALYs. Journal of Health Economics 23, 353-367.

[13] Doctor, J., Miyamoto, J., Bleichrodt H., 2009. When are person tradeoffs valid? Journal of Health Economics 28, 1018-1027.

[14] Dolan, P., 1998. The measurement of individual utility and social welfare. Journal of Health Economics 17, 39-52.

[15] Dolan, P., 2000. The measurement of health-related quality of life, in Culyer, A.J., Newhouse, J.P. (Eds.), Handbook of Health Economics. Elsevier Science, North-Holland, Amsterdam.

[16] Fleurbaey, M., Maniquet, F., 2011, A Theory of Fairness and Social Welfare, Econometric Society Monograph, Cambridge University Press.

[17] Fleurbaey, M., Schokkaert, E., 2009. Unfair inequalities in health and health care. Journal of Health Economics 28, 73-90.

[18] Gafni, A., Birch, S., Mehrez, A., 1993. Economics, health and health economics: HYEs versus QALYs, Journal of Health Economics 12, 325-339.

[19] Gold, M., Siegel, J., Rusell, L., Weinstein, M., 1996, Cost-effectiveness in Health and Medicine, Oxford University Press, New York.

[20] Harvey, C. M. and L.P. Østerdal (2010), Cardinal scales for health evaluation, Decision Analysis, 7, 256-281.

[21] Loomes, G., McKenzie, L., 1989, The use of QALYs in health care decision making. Social Science and Medicine 28, 299-308.

[22] Mehrez, A., Gafni, A., 1989, Quality-adjusted life years, utility theory, and healthy years equivalents. Medical Decision Making 9, 142-149. 
[23] Miyamoto, J., Wakker, P., Bleichrodt, H., Peters, H., 1998, The zero-condition: A simplifying assumption in QALY measurement and multiattribute utility, Management Science 44, 839-849.

[24] Moulin, H., 1988. Axioms of cooperative decision making. Cambridge University Press.

[25] Murray C., Salomon J., Mathers C., 2002, A critical examination of summary measures of population health, in Murray C., Salomon J., Mathers C., Lopez A., (Eds.) Summary Measures of Population Health: Concepts, Ethics, Measurement and Application. World Health Organization.

[26] Pliskin, J., Shepard, D., Weinstein, M., 1980. Utility functions for life years and health status. Operations Research 28, 206-224.

[27] Samuelson, P.A., 1965. Using full duality to show that simultaneously additive direct and indirect utilities implies unitary price elasticity of demand. Econometrica 33, 781-796

[28] Spencer, A., 2003, A test of the QALY model when health varies over time, Social Science and Medicine 57, 1697-1706

[29] Thomson, W., 2001, On the axiomatic method and its recent applications to game theory and resource allocation. Social Choice and Welfare 18, 327-386.

[30] Torrance, G., 1976, Health Status Index Models: A Unified Mathematical View. Management Science 22, 990-1001

[31] Torrance, G., 1986, Measurement of Health State Utilities for Economic Appraisal. A Review. Journal of Health Economics 5, 1-30.

[32] Turpcu, A., Bleichrodt, H., Le, Q., Doctor J., 2011. How To Aggregate Health? Separability and the Effect of Framing. Medical Decision Making. Forthcoming.

[33] Wagstaff A., 1991, QALYs and the equity-efficiency trade-off. Journal of Health Economics $10,21-41$.

[34] Wakker, P., 1996. A criticism of healthy-years equivalents. Medical Decision Making 16, 207-214.

[35] Williams, A., 1997. Intergenerational equity: an exploration of the 'fair innings' argument. Health Economics 6, 117-132. 
[36] Østerdal L.P., 2005, Axioms for health care resource allocation. Journal of Health Economics 24, 679-702. 